\title{
GMR
}

\section{Nuclear magnetic resonance-based study reveals the metabolomics profile of nasopharyngeal carcinoma}

\author{
Y. Wang ${ }^{1}$, X. Luo ${ }^{2}$, G.H. Zhang ${ }^{1}$ and S.L. Li ${ }^{1}$ \\ ${ }^{1}$ Department of Radiation Medicine, Chongqing Medical University, \\ Chongqing, China \\ ${ }^{2}$ Department of Preventive Medicine, North Sichuan Medical College, \\ Nanchong, Sichuan, China \\ Corresponding author: S.L. Li \\ E-mail: shaolin_li@fudedu.com \\ Genet. Mol. Res. 15 (2): gmr. 15027796 \\ Received December 17, 2015 \\ Accepted January 15, 2016 \\ Published May 23, 2016 \\ DOI http://dx.doi.org/10.4238/gmr.15027796
}

\begin{abstract}
Proton nuclear magnetic resonance ([$\left.\left.{ }^{1} \mathrm{H}\right]-\mathrm{NMR}\right)$ spectroscopy has been used to investigate metabolites in serum and several types of tissue. We used NMR spectroscopy to explore the differential metabolic profiles in serum from nasopharyngeal carcinoma (NPC) patients. Moreover, metabolites with potential as biomarkers for identifying NPC patients were primarily identified. Serum samples were collected from 40 enrolled participants comprising 20 healthy subjects and 20 NPC patients. Samples were analyzed using a $600-\mathrm{MHz}$ NMR spectrometer. The $\left[{ }^{1} \mathrm{H}\right]-\mathrm{NMR}$ spectra were further analyzed with partial least squares-discriminant analysis for screening differential metabolites. NMR spectroscopy identified a total of eight metabolites that were present at different levels when the sera of NPC patients were compared with those of healthy individuals. Methionine, taurine $(\mathrm{P}<$ $0.05)$, and choline-like metabolites $(\mathrm{P}<0.05)$ were mostly elevated in the sera of NPC patients. In contrast, the levels of lipids $(\mathrm{P}<0.01)$, isoleucine $(\mathrm{P}<0.05)$, unsaturated lipids $(\mathrm{P}<0.01)$, trimethylamine
\end{abstract}


oxidase $(\mathrm{P}<0.05)$, and carbohydrates $(\mathrm{P}<0.05)$ were lower in the sera of the NPC patients than in the healthy controls. We explored the differential metabolic profiles in sera from NPC patients. $\left[{ }^{1} \mathrm{H}\right]-\mathrm{NMR}$ spectroscopy can be used to identify specific metabolites, and is capable of distinguishing between NPC patients and healthy individuals.

Key words: Nasopharyngeal carcinoma; Metabolomics; NMR spectroscopy

\section{INTRODUCTION}

Nasopharyngeal carcinoma (NPC) is one of the most common malignant tumors, with an annual incidence of 20-50 per 100,000 people in China. Over 650,000 people die of NPC each year worldwide (Jemal et al., 2011). NPC is characterized by radiosensitive tumors and has traditionally been treated by radiotherapy (Mei et al., 2007). However, local relapses and distant metastases frequently occur after radiotherapy alone (Wu et al., 2014). Concurrent chemoradiotherapy and the combination with adjuvant chemotherapy significantly prolongs the survival time of NPC patients (Liu et al., 2013). Although recent medical advances have extended the 5-year overall survival rate, there is still no marker for NPC prognosis, except for disease stage (Chen et al., 2014). Unfortunately, the majority of NPC patients in endemic areas are diagnosed at an advanced stage, owing to the relatively inaccessible nasopharynx site and the insidious clinical course of NPC in China (Liu et al., 2013). The development of novel serum biomarkers that could act as a surrogate for clinical examination would allow the detection of NPC at an earlier stage. This would facilitate proper treatment for NPC patients and significantly improve the overall survival rate. In addition, it would allow blood-based monitoring of the patient's condition during the course of the treatment.

Metabolomics is a rapidly evolving field that aims to quantify the concentration of numerous metabolites and evaluate changes in metabolite levels in body fluids (e.g., blood, saliva, urine) (Larive et al., 2015). Abnormal variations of metabolites have been associated with the biochemical status of organisms (Clayton et al., 2006; Holmes et al., 2008). Thus, the changes in metabolites arising from pathological processes, such as carcinogenesis, and the response to chemotherapy have been investigated by metabolomic methods (Denkert et al., 2006). Metabolomics technology has the potential to measure hundreds of small molecules in a biospecimen, and is obviously superior to traditional approaches that characterize just a single index (Goedert et al., 2014).

Nuclear magnetic resonance (NMR) has emerged as a highly promising technique in cancer research, offering an entirely novel tool for developing metabolomics profiles from tumor samples (Mirnezami et al., 2014). NMR spectroscopy offers several advantages; it is rapid, non-destructive, non-biased, non-invasive, and requires little or no sample preparation. Thus, NMR can provide tremendous amounts of information on the identity and quantity of a large number of metabolites from specimens (Gowda et al., 2008). It will enable better understanding of the molecular mechanisms that regulate disease state and allow the clinician to monitor the response to certain therapies (Gowda et al., 2008). To date, few studies have revealed the metabolic profiles in NPC serum or explored metabolic biomarkers for the diagnosis of NPC. We therefore conducted the present study to compare differential proton nuclear magnetic resonance $\left(\left[{ }^{1} \mathrm{H}\right]-\mathrm{NMR}\right)$ spectra from the serum metabolites of NPC patients 
with those from healthy controls. Partial least squares-discriminant analysis (PLS-DA) identified eight distinguishing metabolites of NPC. The results showed that NPC patients can be diagnosed based on those differential metabolites, which can be used as parameters for monitoring disease. In addition, the data may provide mechanistic profiles of NPC that reflect the novel dysregulation mechanism during NPC tumorigenesis.

\section{MATERIAL AND METHODS}

\section{Instruments and reagents}

All samples were processed in a TGL16G desktop high-speed centrifuge (Anting Scientific Instrument, Shanghai, China) and analyzed on a $600-\mathrm{MHz}$ NMR spectrometer (Japanese Electronics, Tokyo, Japan). Deuterium oxide $\left(\mathrm{D}_{2} \mathrm{O}\right)$ was purchased from Cambridge

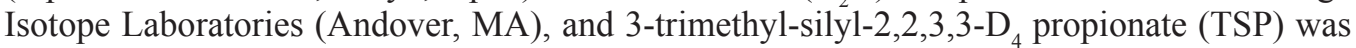
purchased from Sigma-Aldrich (Saint Louis, MO, USA).

\section{Study populations and sample collection}

A total of 20 male patients with NPC and 20 healthy controls (without a history of NPC) were recruited at the First Affiliated Hospital of North Sichuan Medical College (Nanchong, China). The mean age of the participants was 59.15 (range, 43-80 years old; Table 1). The healthy controls comprised 13 male and 7 female subjects with a mean age of 52.6 years. Blood samples $(5 \mathrm{~mL})$ were collected from the 20 NPC patients (the First Affiliated Hospital of North Sichuan Medical College) and the 20 healthy controls, and stored at $-80^{\circ} \mathrm{C}$ until required. Serum was clotted for $1 \mathrm{~h}$ at room temperature, and then centrifuged at 6000 $\mathrm{rpm}$ for $10 \mathrm{~min}$ at $4^{\circ} \mathrm{C}$. All subjects had fasted for $12 \mathrm{~h}$ and refrained from eating irritating food one day before blood collection. There were no additional limitations on variables such as lifestyle or menstrual cycle. The study protocol was approved by the Ethics Committee of the First Affiliated Hospital of North Sichuan Medical College (Nanchong, China). Table 1 outlines the characteristics of the NPC patients studied in this study.

\section{Sample pretreatment and NMR experimental protocol}

All serum samples were thawed and centrifuged at $12,000 \mathrm{~g}$ for $10 \mathrm{~min}$ at $4{ }^{\circ} \mathrm{C}$. The supernatants were placed in $5-\mathrm{mL}$ NMR tubes and mixed with $0.35 \mathrm{~mL}$ phosphate buffer $(0.2$ $\mathrm{M}, \mathrm{pH}$ 7.4) and $0.1 \mathrm{~mL} \mathrm{D}_{2} \mathrm{O}$ (containing $1.5 \mathrm{mM}$ TSP). The treated serum samples were then transferred to 5-mL NMR tubes and investigated using a 600-MHz NMR spectrometer. Onedimensional $\left[{ }^{1} \mathrm{H}\right]$ Carr-Purcell-Meiboom-Gill (CPMG) spectra were acquired using a Bruker Avance III 400-MHz spectrometer equipped with a magic angle-spinning probe (Bruker BioSpin GmbH, Rheinstetten, Germany). All NMR spectra were recorded at $27^{\circ} \mathrm{C}$. Protein signals arising from serum were suppressed by $\mathrm{CPMG}$ pulse sequences. A water suppression pulse was applied during experimentation to minimize the water signal, and the saturation time was $2 \mathrm{~s}$ (Nicholson et al., 1995). With a spin-spin relaxation delay of $3 \mathrm{~s}$ and a spectral width of 10,000 Hz, each NMR spectrum recorded 128 free induction decay (FID) and zero-filled to $64 \mathrm{~K}$ data points. All spectra acquired were phase- and baseline-corrected, and referenced a methyl peak of TSP (d0.0). 
Table 1. Clinical characteristics of nasopharyngeal carcinoma (NPC) patients.

\begin{tabular}{l|l|l|l|c}
\hline Number & Age & Gender & Histopathological classification & TNM staging \\
\hline 1 & 45 & Male & Poorly differentiated SCC & T2N1M0 \\
\hline 2 & 67 & Male & Poorly differentiated SCC & T3N2M0 \\
\hline 3 & 52 & Male & Poorly differentiated SCC & T2N2M0 \\
\hline 4 & 47 & Male & Moderately differentiated SCC & T1N0M0 \\
\hline 5 & 72 & Male & Poorly differentiated SCC & T2N1M0 \\
\hline 6 & 43 & Male & Poorly differentiated SCC & T2N2M0 \\
\hline 7 & 65 & Male & Moderately differentiated SCC & T1N1M0 \\
\hline 8 & 55 & Male & Poorly differentiated SCC & T2N2M0 \\
\hline 9 & 70 & Male & Poorly differentiated SCC & T1N1M0 \\
\hline 10 & 63 & Male & Moderately differentiated SCC & T3N2M0 \\
\hline 11 & 64 & Male & Moderately differentiated SCC & T1N1M0 \\
\hline 12 & 57 & Male & Poorly differentiated SCC & T2N2M0 \\
\hline 13 & 53 & Male & Poorly differentiated SCC & T2N1M0 \\
\hline 15 & 58 & Male & Poorly differentiated SCC & T2N1M0 \\
\hline 16 & 61 & Male & Poorly differentiated SCC & T1N0M0 \\
\hline 17 & 63 & Male & Poorly differentiated SCC & T2N1M0 \\
\hline 18 & 50 & Male & Poorly differentiated SCC & T3N2M0 \\
\hline 19 & 80 & Male & Poorly differentiated SCC & T2N1M0 \\
\hline 20 & 56 & Male & Moderately differentiated SCC & T2N1M0 \\
\hline SCC & 62 & Male & Poorly differentiated SCC &
\end{tabular}

$\mathrm{SCC}=$ squamous cell carcinoma.

\section{NMR data analysis}

To decrease differences in chemical shift caused by the $\mathrm{pH}$ and ions from the serum, the NMR spectra (d10.0-0.0 ppm) were divided into 250 segments of an equal width of d0.04 ppm using the MestReNova 6.1 software prior to pattern recognition and analysis, and each segment was integrated. The region of $\delta 5.2-4.7 \mathrm{ppm}$ was defined as " 0 " owing to spectral difference caused by water suppression (Gavaghan et al., 2000). To reduce the error produced by differences in the concentration of the samples, the integral value for each segment was normalized to the total sum of the spectral intensity, and the normalized integral value was transformed to Excel data format for analysis using SIMCA-P10.5 (Umetrics, Umea, Sweden) and PLS-DA (Cheng et al., 1998).

\section{Statistical analysis}

The NMR data, normalized by dividing the sum of all peak areas in the sample (1 mg) before multivariate analysis, are reported as means \pm SD and were introduced into SPSS 17.0 (SPSS Inc., Chicago, IL, USA) for Windows. The differences in concentration between the two panels of metabolites were compared by the independent $t$-test. A P value less than 0.05 was considered statistically significant. PLS-DA was performed to differentiate the metabolic patterns in the two different groups.

\section{RESULTS}

\section{Metabolic profiling in NPC patients and healthy controls}

Typical $\left[{ }^{1} \mathrm{H}\right]-\mathrm{NMR}$ spectra of serum from NPC patients and healthy controls are described in Figure 1. The standard one-dimension spectrum gave an overview of all 
metabolites. The major metabolites in the spectra were identified according to literature data and the Human Metabolome Database (http://www.hmdb.ca/) (Wang et al., 2013). As a result, a series of differential metabolite levels were observed in the plasma from NPC patients in comparison with healthy controls. Individual metabolites were identified using NMR databases. As shown in Figure 1, serum levels of isoleucine (d0.93), valine (d0. 99, d1.04), lactate (d1.33, 84.12), alanine (d1.48), N-acetyl glycoproteins N (NAC, d2.04), methionine (d2.13), acetoacetate $(\mathrm{d} 2.22)$, pyruvate $(\mathrm{d} 2.37)$, glutamine $(\mathrm{d} 2.41)$, creatine (d3.03), phosphocholine/ choline (d3.22), trimethylamine oxidase (TMAO) (d3.27), and glucose (d3.70-3.90) were significantly different in the plasma samples from the NPC patients compared with those from the healthy controls.

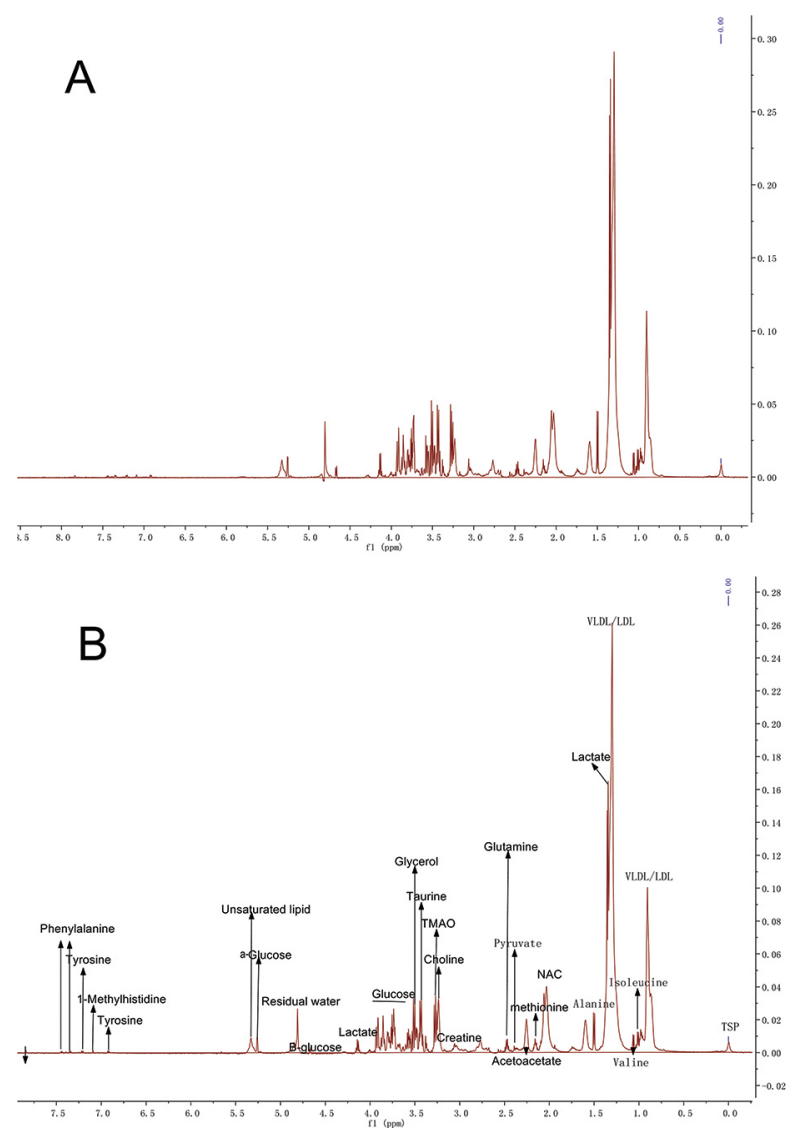

Figure 1. $600 \mathrm{MHz}$ representative proton nuclear magnetic resonance $\left(\left[{ }^{1} \mathrm{H}\right]-\mathrm{NMR}\right)$ spectra of serum samples. A. Nasopharyngeal carcinoma (NPC) patients; B. healthy controls.

\section{Profiles of metabolites for distinguishing between NPC patients and healthy controls}

To further distinguish NPC patients from healthy controls based on metabolic profiles, we then utilized PLS-DA to visualize the differential metabolic profiles. The data are represented by the PLS-DA loading plot (Figure 2A) and the scores plot (Figure 2B). The 
PLS-DA loading plot represents integral segments, whereas each score shows the value of each serum sample. The distinction of differential metabolites usually depends on the distance of the peak spectral region from zero. A metabolite was selected when the distance of its peak spectral region from zero differed between samples. Thus, analysis of the PLS-DA loading plot and the scores plot enabled us to explore differential metabolites in the serum samples from NPC patients and healthy controls. In Figure 2B, the PLS-DA scores plot indicates that the serum of NPC patients had significantly different metabolite profiles, and it can be used to distinguish NPC patients from healthy subjects.
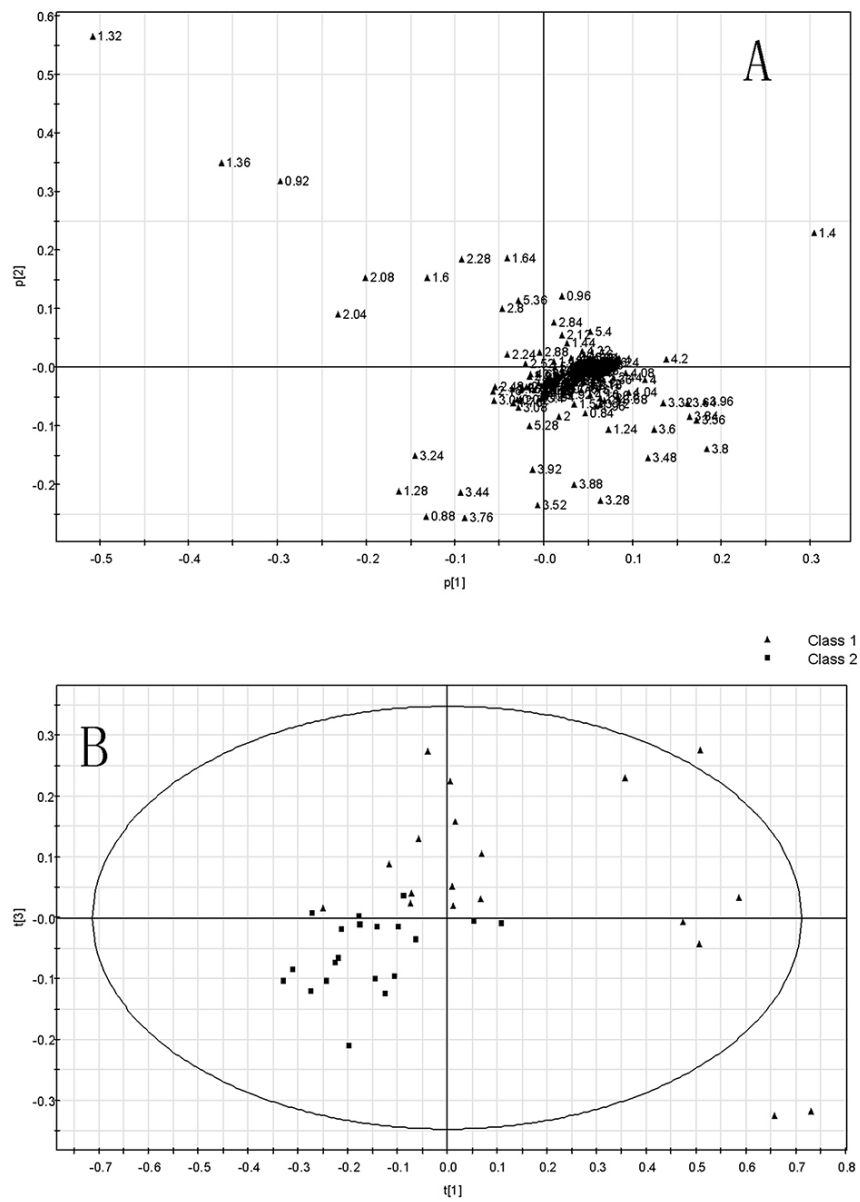

Figure 2. Partial least squares-discriminant analysis (PLS-DA) loading plot (A) and scores plot (B) of serum samples from healthy controls (filled triangles) and nasopharyngeal carcinoma (NPC) patients (filled squares).

As shown in Table 2, the levels of methionine, taurine, and choline-like metabolites in the serum from NPC patients were higher than those from the healthy controls. In contrast, there were lower levels of lipids, isoleucine, unsaturated lipids, TMAO, and carbohydrates in the serum from the NPC patients compared with the healthy controls. 
Table 2. Comparison of metabolite levels in serum from nasopharyngeal carcinoma (NPC) patients and healthy controls.

\begin{tabular}{l|c|c|c|c}
\hline Group & Glucose & Isoleucine & Methionine & LDL \\
\hline Healthy controls & $12.9 \pm 3.1$ & $7.0 \pm 2.4$ & $1.2 \pm 0.5$ & $40.1 \pm 15.3$ \\
\hline NPC patients & $6.1 \pm 1.2^{* *}$ & $3.8 \pm 1.2^{*}$ & $4.2 \pm 1.2^{*}$ & $25.1 \pm 13.6^{*}$ \\
\hline & Choline & TMAO & Taurine & Lipids \\
\hline Healthy controls & $8.1 \pm 4.8$ & $9.2 \pm 5.1$ & $6.9 \pm 3.2$ & $10.1 \pm 7.1$ \\
\hline NPC patients & $14.5 \pm 7.6^{*}$ & $3.4 \pm 1.6^{*}$ & $10.2 \pm 5.3^{*}$ & $1.8 \pm 0.4^{* *}$ \\
\hline
\end{tabular}

$\mathrm{LDL}=$ low-density lipoprotein; $\mathrm{TMAO}=$ trimethylamine oxidase. $* \mathrm{P}<0.05 ; * * \mathrm{P}<0.01$.

\section{DISCUSSION}

NMR is a high-throughput technique that is characterized by high resolution and sensitivity. It has been widely used to explore metabolic profiling in various biological samples. As mentioned above, NMR spectroscopy is one of the premier methods because it has several advantages; it is rapid, non-destructive, and non-invasive (Gowda et al., 2008). In the present study, we investigated the metabolomics of NPC patients by exploring metabolic biomarkers for the diagnosis of NPC. A series of metabolites with differential levels, such as isoleucine and glucose, were identified in the serum of NPC patients. Those metabolites were selected based on NMR spectra and were analyzed by PLS-DA.

Lipids were present at lower levels in the serum from NPC patients in our study. Disorders of lipid metabolism cause decreases of lipid concentration, and increase the lipid clearance capability of tumors (Cheng et al., 1998). For instance, cholesterol is present in remarkably high levels in tumor tissues. The reason for this might be that cholesterol, combined with lowdensity lipoprotein (LDL), is transported into the cytoplasm of tumor cells. Subsequently, the cholesterol is rapidly degraded. As a result, cholesterol and LDL levels are reduced in the serum of NPC patients (Shi et al., 2011). As an essential amino acid, methionine is necessary for protein synthesis. Lack of methionine restricts protein synthesis (Hazari et al., 2015). Methionine can resist membrane lipid peroxidation mediated by oxygen free radicals in several ways (Jung et al., 2013). Lipid peroxidation damages the membranes of primary and secondary lysosomes and releases lysosomal acid phosphatase (Skibba et al., 1986). The increased levels of serum methionine in NPC patients indicate an association with reduced oxygen free radicals. The NPC metabolic profiles in the present study revealed that there were higher levels of methionine in the NPC serum, suggesting that it might play an important role in regulating the metabolism of NPC. Furthermore, metabolites of choline such as phosphatidylcholine are components of the membrane and phospholipoproteins, and play an essential role in lipid metabolism and membrane integrity; they were present at high levels in the serum from NPC patients, and the increase of choline in tumor cells might destroy membrane permeability, resulting in damage to cell structure (Cheng et al., 1998; Yang et al., 2007).

Here, we showed that the concentrations of glucose and lactic acid were reduced in the serum of NPC patients compared with the healthy controls. These findings are consistent with studies in breast cancer, which also demonstrated that those compounds were present at lower levels in patients with breast cancer (Whitehead and Kieber-Emmons, 2005). The dysregulation of those metabolic compounds is caused by the abnormal glucose metabolism in cancer tissues, as described in Warburg's theory about the major source of ATP from glucose fueling cellular proliferation (the Warburg effect) (Weinberg et al., 2010; Hanahan 
and Weinberg, 2011). In the absence of oxygen, glucose is converted to lactic acid during glycolysis. However, dysregulation in glucose metabolism in NPC patients may result in increased glycolysis. This phenomenon has been observed in liver cancer where glucose concentration is reduced, while lactic acid is elevated in NPC patients (Cheng et al., 1998). These observations might be due to cellular proliferation and the subsequently increased energy demand. These processes consume more glucose for the production of energy, the promotion of glycolysis, and the accumulation of lactic acid (Weinberg et al., 2010). The liver is the major organ for the conversion and metabolism of lactic acid, which enters the liver from the blood stream and eventually breaks down into water and carbon dioxide. Therefore, lactic acid does not accumulate in the blood (Cheng et al., 1998). Dynamic metabolic profiling using ${ }^{1}$ NMR provides clues about biochemical pathways for the diagnosis of NPC, which could help clinicians determine the most appropriate treatment for NPC patients.

In summary, we investigated serum metabolomics in NPC patients using NMR spectra. Our results suggest that the metabolism of glucose, lipids, and protein is associated with the pathogenesis of NPC. Further studies are needed for a better understanding of how these metabolic pathways are regulated in healthy people and in NPC patients.

\section{Conflicts of interest}

The authors declare no conflict of interest.

\section{ACKNOWLEDGMENTS}

Research supported by the Natural Science Foundation of China (Foundation \#81171365).

\section{REFERENCES}

Chen Y, Li X, Wu S, Xu G, et al. (2014). Expression of HIF-1 $\alpha$ and CAIX in nasopharyngeal carcinoma and their correlation with patients' prognosis. Med. Oncol. 31: 304-310. http://dx.doi.org/10.1007/s12032-014-0304-1

Cheng LL, Chang IW, Smith BL and Gonzalez RG (1998). Evaluating human breast ductal carcinomas with highresolution magic-angle spinning proton magnetic resonance spectroscopy. J. Magn. Reson. 135: 194-202. http:// dx.doi.org/10.1006/jmre.1998.1578

Clayton TA, Lindon JC, Cloarec O, Antti H, et al. (2006). Pharmaco-metabonomic phenotyping and personalized drug treatment. Nature 440: 1073-1077. http://dx.doi.org/10.1038/nature04648

Denkert C, Budczies J, Kind T, Weichert W, et al. (2006). Mass spectrometry-based metabolic profiling reveals different metabolite patterns in invasive ovarian carcinomas and ovarian borderline tumors. Cancer Res. 66: 10795-10804. http://dx.doi.org/10.1158/0008-5472.CAN-06-0755

Gavaghan CL, Holmes E, Lenz E, Wilson ID, et al. (2000). An NMR-based metabonomic approach to investigate the biochemical consequences of genetic strain differences: application to the C57BL10J and Alpk:ApfCD mouse. FEBS Lett. 484: 169-174. http://dx.doi.org/10.1016/S0014-5793(00)02147-5

Goedert JJ, Sampson JN, Moore SC, Xiao Q, et al. (2014). Fecal metabolomics: assay performance and association with colorectal cancer. Carcinogenesis 35: 2089-2096. http://dx.doi.org/10.1093/carcin/bgu131

Gowda GA, Zhang S, Gu H, Asiago V, et al. (2008). Metabolomics-based methods for early disease diagnostics. Expert Rev. Mol. Diagn. 8: 617-633. http://dx.doi.org/10.1586/14737159.8.5.617

Hanahan D and Weinberg RA (2011). Hallmarks of cancer: the next generation. Cell 144: 646-674. http://dx.doi. org/10.1016/j.cell.2011.02.013

Hazari PP, Prakash S, Meena VK, Jaswal A, et al. (2015). LAT1 targeted delivery of methionine based imaging probe derived from $\mathrm{M}(\mathrm{III})$ metal ions for early diagnosis of proliferating tumours using molecular imaging modalities. Curr. Cancer Drug Targets 14: 817-831.http://dx.doi.org/10.2174/1568009614666141020102337 
Holmes E, Wilson ID and Nicholson JK (2008). Metabolic phenotyping in health and disease. Cell 134: 714-717. http:// dx.doi.org/10.1016/j.cell.2008.08.026

Jemal A, Bray F, Center MM, Ferlay J, et al. (2011). Global cancer statistics. CA Cancer J. Clin. 61: 69-90. http://dx.doi. org/10.3322/caac.20107

Jung HJ, Kim HL and Seo YR (2013). Enhanced redox factor 1 (REF1)-modulated p53 stabilization and JNK1 dissociation in response to selenomethionine. Anticancer Res. 33: 3645-3651.

Larive CK, Barding GA, Jr. and Dinges MM (2015). NMR spectroscopy for metabolomics and metabolic profiling. Anal. Chem. 87: 133-146.http://dx.doi.org/10.1021/ac504075g

Liu Q, Chen JO, Huang QH and Li YH (2013). Trends in the survival of patients with nasopharyngeal carcinoma between 1976 and 2005 in Sihui, China: a population-based study. Chin J Cancer 32: 325-333. http://dx.doi.org/10.5732/ cjc. 012.10189

Mei YP, Zhou JM, Wang Y, Huang H, et al. (2007). Silencing of LMP1 induces cell cycle arrest and enhances chemosensitivity through inhibition of AKT signaling pathway in EBV-positive nasopharyngeal carcinoma cells. Cell Cycle 6: 1379-1385.http://dx.doi.org/10.4161/cc.6.11.4274

Mirnezami R, Jiménez B, Li JV, Kinross JM, et al. (2014). Rapid diagnosis and staging of colorectal cancer via highresolution magic angle spinning nuclear magnetic resonance (HR-MAS NMR) spectroscopy of intact tissue biopsies. Ann. Surg. 259: 1138-1149. http://dx.doi.org/10.1097/SLA.0b013e31829d5c45

Nicholson JK, Foxall PJ, Spraul M, Farrant RD, et al. (1995). $750 \mathrm{MHz} 1 \mathrm{H}$ and 1H-13C NMR spectroscopy of human blood plasma. Anal. Chem. 67: 793-811. http://dx.doi.org/10.1021/ac00101a004

Shi HB, Jiang JT and Wu CP (2011). Clinical significance of changes in lipids and total cholesterol in patients with gastric cancer. Chin. Clin. Oncol. 16: 686-688.

Skibba JL, Quebbeman EJ and Kalbfleisch JH (1986). Nitrogen metabolism and lipid peroxidation during hyperthermic perfusion of human livers with cancer. Cancer Res. 46: 6000-6003.

Wang H, Wang L, Zhang H, Deng P, et al. (2013). ${ }^{1} \mathrm{H}$ NMR-based metabolic profiling of human rectal cancer tissue. Mol. Cancer 12: 121-133. http://dx.doi.org/10.1186/1476-4598-12-121

Weinberg F, Hamanaka R, Wheaton WW, Weinberg S, et al. (2010). Mitochondrial metabolism and ROS generation are essential for Kras-mediated tumorigenicity. Proc. Natl. Acad. Sci. USA 107: 8788-8793. http://dx.doi.org/10.1073/ pnas. 1003428107

Whitehead TL and Kieber-Emmons T (2005). Applying in vitro NMR spectroscopy and 1H NMR metabonomics to breast cancer characterization and detection. Prog. Nucl. Magn. Reson. Spectrosc. 47: 165-174. http://dx.doi.org/10.1016/j. pnmrs.2005.09.001

Wu F, Wang R, Lu H, Wei B, et al. (2014). Concurrent chemoradiotherapy in locoregionally advanced nasopharyngeal carcinoma: treatment outcomes of a prospective, multicentric clinical study. Radiother. Oncol. 112: 106-111.http:// dx.doi.org/10.1016/j.radonc.2014.05.005

Yang C, Richardson AD, Smith JW and Osterman A (2007). Comparative metabolomics of breast cancer. Pac. Symp. Biocomput. 2007: 181-192. 